Rev. Biol. Neotrop. 8(2): 1-12. 2011

\title{
ARIAÇÕES AMBIENTAIS E RELAÇÕES FLORÍSTICAS NO CERRADO SENSU STRICTO SOBRE AREIA (NEOSSOLO QUARTZARÊNICO) da Chapada Grande Meridional, Piauí
}

\author{
GALIANA DA SILVEIRA LINDOSO
}

Programa de Pós-graduação em Ecologia, Universidade de Brasília; e-mail: gali.lindoso@ gmail.com

\section{JEANINE MARIA FELFILI}

Departamento de Engenharia Florestal, Universidade de Brasília; in memoriam

\section{LUCAS DE CARVALHO RAMOS SILVA}

Departamento de Engenharia Florestal, Universidade de Brasília (durante realização do trabalho); Dept. Land, Air and Water Resources, University of California Davis (atual); e-mail: lucascrsilva@gmail.com

\begin{abstract}
RESUMO: O presente estudo teve como objetivo relacionar a variação florística e estrutural do cerrado sensu stricto sobre Neossolo Quartzarênico na Chapada Grande Meridional, que ocorre em manchas em uma região ecotonal, com a variação das características edáficas e da altitude. Os teores de nutrientes e os valores de pH obtidos para os solos da Chapada Grande Meridional encontram-se no intervalo comumente observado em solos de cerrado, caracterizando-os como ácidos, distróficos e com textura arenosa. Na Análise de Correspondência Canônica (CCA) as variáveis ambientais que tiveram maior influência na vegetação foram: altitude e teores de $\mathrm{Na}^{+}, \mathrm{K}^{+}, \mathrm{Al}^{3+}, \mathrm{P}^{+}, \mathrm{Zn}^{2+}$ e areia grossa. A CCA mostrou gradientes de fertilidade, altitude, textura e salinidade do solo que influenciaram na densidade de espécies, caracterizando uma elevada diversidade beta em um mesmo tipo de solo.
\end{abstract}

Palavras-chave: Nordeste, ordenação, savana, solos.

ABSTRACT: In this study we investigated the relationship between the floristic and structure variations of the cerrado sensu stricto (savanna woodland physiognomy) that occur on Entisols (Quartzarenic Neosols) regarding the soils characteristics and altitude. At Chapada Grande Meridional, the gradient of fertility and $\mathrm{pH}$ occurs in an interval commonly found in soils of cerrado, characterized as acids, dystrophic and with a sandy texture. At the Canonical Correspondence Analysis (CCA) the environmental variables that most influenced the vegetation were altitude, quantities of $\mathrm{Na}^{+}, \mathrm{K}^{+}, \mathrm{Al}^{3+}, \mathrm{P}^{+}, \mathrm{Zn}^{2+}$ and coarse sand. The gradients of fertility, altitude, texture and salinity of the soil influenced the density of some species, characterizing elevated beta diversity even in a same kind of soil.

KEY-WORDS: Northeast, ordination, savannas, soils.

\section{INTRODUÇÃO}

$\mathrm{O}$ cerrado sensu stricto (s.s.), fitofisionomia que cobre cerca de $70 \%$ do bioma Cerrado (Eiten, 1972), é caracterizado por possuir um padrão de distribuição das espécies lenhosas em mosaico, onde apesar da homogeneidade estrutural existe grande variação na composição florística e densidade de espécies (Felfili \& Felfili, 2001; Felfili et al., 2004, 2007; Felfili \& Silva Júnior, 1993, 2001, 2005; Ratter et al. 2003, 2005).
Estudos anteriores demonstram que variações no ambiente edáfico, como na disponibilidade de nutrientes, de matéria orgânica e diferenças na textura dos solos, podem ocasionar essas diferenças na vegetação (Goodland \& Pollard, 1973; Haridasan, 1992; MarimonJunior \& Haridasan, 2005; Oliveira-Filho et al., 1989; Ruggiero et al., 2002). Contudo, no bioma Cerrado, a maior parte dos estudos realizados é restrita às áreas de Latossolos, que são os solos predominantes no bioma e poucos são os trabalhos que contemplam as relações entre as 
variações florísticas em solos arenosos, como o Neossolo Quartzarênico (Haridasan, 2001, 2007; Lindoso \& Felfili, 2007), que corresponde a $15,2 \%$ da extensão do bioma (Reatto \& Martins, 2005).

O Neossolo Quartzarênico, também conhecido como Areia Quartzosa, caracteriza-se por ser bem-drenado, ácido, com baixa fertilidade e alta saturação por alumínio, além de apresentar pouca diferenciação nos perfis, que são em geral profundos, com no mínimo dois metros de profundidade (Furley \& Ratter, 1988; Goedert, 1987). Possui no máximo 15\% de argila, com textura classificada como arenosa à franco-arenosa (Embrapa, 1999; Reatto et al., 1998), com severa limitação na capacidade de armazenamento de água (Reatto et al., 1998; Sousa \& Lobato, 2005) e reduzida capacidade de agregação de partículas e adsorção de nutrientes, atribuída aos baixos teores de argila e matéria orgânica, o que torna o solo muito suscetível à erosão e à perda de nutrientes por lixiviação (Reatto et al., 1998).

Nessas condições de escassos recursos, espera-se que o estabelecimento de desenvolvimento da vegetação lenhosa responda mesmo à pequenas variações nos atributos edáficos. Extensas áreas de Neossolo Quartzarênico ocorrem no Mato Grosso do Sul, Mato Grosso, oeste da Bahia, sul do Maranhão, norte e sul do Piauí, norte de Minas Gerais e sul do Pará (Goedert, 1987; Reatto et al., 1998), onde mudanças na vegetação de cerrado s.s. está, aparentemente, associada não somente a um gradiente nos teores de nutrientes disponíveis e pH dos solos (Haridasan, 2001), mas também à influência das diferenças do regime de água nos solos, declividade e rochosidade (Oliveira-Filho et al., 1989).

No entanto, não somente interações solo-planta são relevantes nesse contexto, mas a flora por si só é elemento de central interesse, uma vez que pouca atenção tem sido devotada aos estudos florísticos nessas regiões. Alguns autores (Castro et al., 1998; Castro \& Martins, 1999) têm ressaltado a necessidade de estudos sobre a composição florística e estrutura da vegetação dos cerrados marginais do Nordeste, assim como estudos para a determinação de padrões biogeográficos relacionando a florística e estrutura da vegetação do Cerrado com as condicionantes ambientais (Silva et al., 2006), especialmente em comunidades encontradas sobre Neossolo Quartzarênico (Haridasan, 2001, 2007; Lindoso \& Felfili, 2007).

Visando, portanto, contribuir ao preenchimento dessas lacunas de conhecimento sobre os cerrados marginais do Nordeste e investigar as relações entre a vegetação e fatores edáficos nas áreas de cerrado estabelecido sobre solos arenosos, o presente estudo teve como objetivo relacionar a variação florística do cerrado s.s. estabelecido sobre Neossolo Quartzarênico, na Chapada Grande Meridional, região ecotonal no Piauí, com variações nas características químicas e físicas do solo e a altitude.

\section{Material e Métodos}

\section{ÁREA DE ESTUDO}

O presente estudo foi conduzido na Chapada Grande Meridional que se localiza na regiãocentraldoPiauí $\left(06^{\circ} 34^{\prime} 45^{\prime \prime}\right.$ Se $\left.42^{\circ} 07^{\prime} 18^{\prime \prime} \mathrm{W}\right)$, na altura setentrional do Rio Parnaíba. O cerrado s.s. sobre Neossolo Quartzarênico na Chapada Grande Meridional é encontrado em manchas, nos municípios de Arraial e Regeneração, intercaladas por outros tipos de vegetação ou solos e, muitas vezes, fragmentadas pelo uso antrópico. Arraial possui uma área de $655 \mathrm{~km}^{2}\left(06^{\circ} 39^{\prime} 18^{\prime \prime} \mathrm{S}\right.$ e $\left.42^{\circ} 31^{\prime} 55^{\prime \prime} \mathrm{W}\right)$ e altitude média de $338 \mathrm{~m}$, enquanto o município de Regeneração possui uma área de 1.266 km² (06 $14^{\circ} 16^{\prime \prime} \mathrm{S}$ e $\left.42^{\circ} 41^{\prime} 18^{\prime \prime} \mathrm{W}\right)$ com altitude média de 164 m (Aguiar \& Gomes, 2004).

O clima na Chapada Grande Meridional é subúmido seco de acordo com a classificação climática de Thornthwaite \& Mather (1955), onde o clima varia de semi-árido a úmido, passando por subúmido seco a Subúmido (Andrade Júnior et al., 2004). O município de Arraial apresenta temperaturas entre $24,1^{\circ} \mathrm{C}$ e $34,3^{\circ} \mathrm{C}$ (média de $27,2^{\circ} \mathrm{C}$ ) e precipitação total anual de 1.109,8 mm, enquanto o município de Regeneração apresenta temperaturas entre $21,7^{\circ} \mathrm{C}$ e $33,2^{\circ} \mathrm{C}$ (média de $26,9^{\circ} \mathrm{C}$ ) e precipitação total anual de aproximadamente $1200 \mathrm{~mm}$ (Lima \& Assunção, 2002).

Os solos da região são provenientes da alteração de arenitos, siltitos, folhelhos, cal- 
cários, basaltos, laterita e gabros, variando entre solos podzólicos com manchas de Neossolo Quartzarênico e Latossolo. O município apresenta formações de cerrado s.s., caatinga, veredas, matas de galeria (Aguiar \& Gomes, 2004) e mata dicótilo-palmácea (que incluem babaçuais, carnaubais e buritizais).

\section{Dados Sobre Vegetação e Variáveis AMBIENTAIS}

Para a coleta de dados sobre a vegetação, foi utilizado o resultado do levantamento fitossociológico realizado nas manchas de cerrado s.s. sobre Neossolo Quartzarênico distribuídas pela Chapada Grande Meridional, com dez parcelas de 20 x 50 m (P1 a P10), totalizando um hectare (maiores detalhes em Lindoso et al., 2009). Em cada parcela, foram coletadas cinco amostras simples de solo, até $15 \mathrm{~cm}$ de profundidade, para compor uma amostra composta por parcela. As amostras foram analisadas nos Laboratórios de Química Analítica da EMBRAPA/CPAC, utilizando-se os métodos descritos pela Embrapa $(1997,2005)$. Foram realizadas as análises de $\mathrm{pH}$, acidez potencial $(\mathrm{H}+\mathrm{Al})$, concentrações disponíveis dos elementos: $\mathrm{Al}^{3+}$, $\mathrm{Ca}^{2+}, \mathrm{Mg}^{2+}, \mathrm{P}^{+}, \mathrm{K}^{+}, \mathrm{Mn}^{2+}, \mathrm{Cu}^{2+}, \mathrm{Zn}^{2+}, \mathrm{Fe}^{2+}, \mathrm{Na}^{+}$ e teor porcentual de matéria orgânica, areia, silte e argila.

As disponibilidades de fósforo, potássio e dos micronutrientes $\left(\mathrm{Cu}^{2+}, \mathrm{Fe}^{2+}, \mathrm{Mn}^{2+}, \mathrm{Zn}^{2+}\right)$ foram determinadas com extração ácida $(0,05$ $\mathrm{M} \mathrm{H}_{2} \mathrm{SO}_{4}+0,05 \mathrm{M} \mathrm{HCl}$ ) e medidas com o espectrofotômetro UV-visível, fotômetro de chama e espectofotômetro de absorção atômica. A extração de $\mathrm{Ca}^{2+}, \mathrm{Mg}^{2+}$ e $\mathrm{Al}^{3+}$ foi feita com solução de $\mathrm{KCl}(1 \mathrm{M})$. O $\mathrm{Al}^{3+}$ foi determinado por titulação ácido-base com $\mathrm{NaOH}(0,01 \mathrm{M})$, e

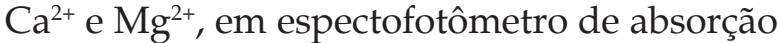
atômica. Para o cálculo da saturação por bases foi utilizada também a acidez potencial $(\mathrm{H}+\mathrm{Al})$ obtida com extração por $\mathrm{C}_{4} \mathrm{H}_{6} \mathrm{CaO}_{4}(0,5 \mathrm{M})$, seguida de titulação ácido-base com $\mathrm{NaOH}(0,02$ M). O percentual de matéria orgânica foi calculado pelo método da oxidação por via úmida (Walkley \& Black, 1934). O pH foi medido em suspensão solo-água $(1: 2,5)$ e a textura foi determinada por diferenças de densidade pelo método da pipeta (Day, 1965). A Capacidade de Troca Catiônica (CTC efetiva e T), a porcentagem de sódio trocável (PST) no solo, saturação de bases trocáveis (V) e saturação por alumínio trocável $(\mathrm{m})$, através das equações (Embrapa, 1997, 2005; Luz et al., 2002):

CTC efetiva $\left(\right.$ cmol. $\left.\mathrm{kg}^{-1}\right)=\mathrm{Na}^{+}+\mathrm{Ca}^{2+}+\mathrm{K}^{+}+$ $\mathrm{Mg}^{2+}+\mathrm{Al}^{3+}$

$\mathrm{T}\left(\mathrm{cmol} . \mathrm{kg}^{-1}\right)=\mathrm{Na}^{+}+\mathrm{Ca}^{2+}+\mathrm{K}^{+}+\mathrm{Mg}^{2+}+\mathrm{H}+\mathrm{Al}$ PST $(\%)=\left(\mathrm{Na}^{+} / \mathrm{CTC}\right.$ efetiva $) \times 100$

$\mathrm{V}(\%)=\left(\mathrm{Na}^{+}+\mathrm{Ca}^{2+}+\mathrm{K}^{+}+\mathrm{Mg}^{2+} / \mathrm{T}\right) \times 100$

$\mathrm{m}(\%)=\left(\mathrm{Al}^{3+} / \mathrm{CTC}\right.$ efetiva $) \times 100$

\section{RELAÇÃo EnTRe VegetaÇÃo e VARIÁVEIS AMBIENTAIS}

As características químicas e físicas do solo, altitude e a densidade absoluta das espécies foram ordenadas por análise direta de gradientes, através da Análise de Correspondência Canônica (CCA) (Ter Braak, 1986) com a utilização do programa CANOCO 4.02 (Ter Braak \& Smilauer, 1999), a fim de detectar relações entre as variáveis ambientais e ocorrência e abundância das espécies nas parcelas. As densidades das espécies foram log-transformadas (Leps \& Smilauer, 2003) e os dados ambientais foram padronizados com base na média e desvio padrão, segundo Ter Braak (1986). Foram utilizadas as espécies que obtiveram densidade maior que 5 indivíduos por hectare na amostragem, totalizando 30 espécies. Após a primeira análise, foram eliminadas as variáveis com alta colinearidade, detectadas pelo alto valor de inflação $(\geq 20,0)$, e as variáveis pouco correlacionadas com os eixos de ordenação, indicado pelo baixo valor dos coeficientes de correlação (menor que 0,3 no primeiro e segundo eixos), totalizando sete variáveis analisadas.

\section{Resultados e Discussão}

\section{VEGETAÇÃO}

No cerrado s.s. sobre Neossolo Quartzarênico da Chapada Grande Meridional foram encontradas 48 espécies e duas subespécies 
distribuídas em 45 gêneros e 26 famílias. A densidade foi de 930 indiv.ha- $^{-1}$, área basal de $12,84 \mathrm{~m}^{2} . \mathrm{ha}^{-1}$ e a diversidade alfa foi de 2,75 nats.indiv ${ }^{-1}$ pelo índice de Shannon (maiores detalhes em Lindoso et al., 2009). Na Tabela 1 estão listadas as 30 espécies que obtiveram densidade maior que 5 indiv. ha ${ }^{-1}$ na amostragem, utilizadas na Análise de Correspondência Canônica (CCA) e indicadas as fisionomias onde ocorrem, além de cerrado s.s., baseado em Castro et al. (1998), Felfili et al. (2001), Mendonça et al. (1998) e Rodal et al. (2005). As espécies analisadas correspondem a $61,22 \%$ das espécies amostradas e a $89,7 \%$ da densidade obtida no levantamento fitossociológico (Lindoso et al., 2009).

As espécies amostradas na Chapada Grande Meridional representam uma mistura de espécies típicas dos cerrados do Nordeste, como Aspidosperma multiflorum, Himatanthus drasticus, Byrsonima crassifolia, Stryphnodendron coriaceum, Parkia platycephala, Caryocar coriaceum, Copaifera coriacea, Combretum leprosum, Combretum mellifluum e Vitex flavens; espécies de ampla distribuição no bioma, como as espécies de Qualea grandiflora, Qualea parviflora e Sclerolobium paniculatum, e que ocorrem na Caatinga, como Cochlospermum regium, Luetzelburgia auriculata e Cenostigma gardneriana (Tabela 1 ).

Qualea grandiflora e Parkia platycephala, que possuíram a maior densidade, correspondem a quase metade dos indivíduos analisados (395). Astronium fraxinifolium, Combretum mellifluum, Vatairea macrocarpa, Eugenia dysenterica e Coccoloba sp, que possuíram as menores densidades (Tabela 1), correspondem a 3,0\%. A concentração da densidade em poucos indivíduos é uma característica marcante do cerrado s.s. (Felfili et al., 2004, 2007; Felfili \& Silva Junior, 2001, 2005) e desta forma, o grupo de espécies com maior densidade define a estrutura da vegetação, de modo que as respostas destas espécies aos parâmetros ambientais tendem a definir as características da comunidade em maior escala.

Das espécies analisadas (Tabela 1), 16 $(53,33 \%)$ possuem ampla distribuição no Cerrado, 63,3\% ocorrem também em fisionomias florestais e 33,3\% também ocorrem na
Caatinga. A relação dinâmica existente entre vegetações florestais e savânicas evidenciadas em escalas locais (Silva et al., 2008) e em escalas regionais desde o Pleistoceno, com a expansão das savanas e contração da Floresta Amazônica nos períodos glaciais e vice-versa, nos períodos interglaciais, promoveu padrões complexos na flora e fragmentação de populações (Oliveira-Filho \& Ratter, 2002; Ratter et al., 1997). Com isso, as formações do bioma, apesar de apresentarem uma flora específica em geral, compartilham espécies das formações savânicas e florestais que se intercalam no espaço geográfico, em função das condicionantes ambientais (Silva et al., 2006; Silva et al., 2008). As espécies florestais e as que ocorrem também em outros biomas adjacentes possuem grande significado ecológico na área de estudo, como demonstrado pelas abundâncias (Tabela 1), corroborando a discussão de Castro \& Martins (1999) \& Ratter et al. (2005), sobre espécies peculiares e não-endêmicas no bioma Cerrado.

\section{Solos}

Apesar dos solos de todas as parcelas amostradas serem ácidos e distróficos em relação ao $\mathrm{Ca}^{2+}$, com concentrações inferiores a 2,0 cmol. $\mathrm{kg}^{-1}$ (Haridasan, 1992; Lopes, 1983) (Tabela 2), os coeficientes de variações obtidos para as variáveis medidas possuem valores moderados a elevados (>20\%), indicando sensíveis variações das propriedades dos solos entre as parcelas.

Em geral os solos possuem baixa capacidade de troca catiônica (CTC menor que 27 cmolc. $\mathrm{kg}^{-1}$ ), refletindo a textura arenosa, com baixa porcentagem de argila, que variou de 3,0 a $6,0 \%$ e baixo teor de matéria orgânica, que variou de $0,21 \%$ a $0,36 \%$ (Tabela 2 ), encontrando-se bem abaixo dos limites considerados típicos para vegetação de cerrado $(0,7$ a 6,0 \%) (Furley \& Ratter, 1988). Contudo, em relação à saturação por bases, as parcelas P1 e P8 mostraram valores superiores a 50\% (Tabela 2) e apenas a $\mathrm{P} 4$ e $\mathrm{P} 9$ possuíram caráter álico, com saturação por $\mathrm{Al}^{3+}$ acima de 50\% (Embrapa, 1999; IBGE 2007). 
Tabela 1 - Espécies do cerrado sensu stricto sobre Neossolo Quartzarênico da Chapada Grande Meridional, Piauí, utilizadas na Análise de Correspondência Canônica, distribuídas em outras fisionomias de ocorrência (baseado em Mendonça et al., 1998; Castro et al., 1998; Felfili et al., 2001; Rodal et al., 2005). MG: mata de galeria; MS: mata seca; Ce: cerradão; C: cerrado sensu stricto; Ca: Caatinga.

\begin{tabular}{|c|c|c|c|}
\hline Espécie & Família & $\mathbf{N}$ & Fitofisionomia \\
\hline Qualea grandiflora Mart. & Vochysiaceae & 280 & $\mathrm{C}$ \\
\hline Parkia platycephala Benth. & Fabaceae & 115 & MG, Ce, C \\
\hline Qualea parviflora Mart. & Vochysiaceae & 70 & MG, C \\
\hline Curatella americana L. & Dilleniaceae & 34 & $\mathrm{Ce}, \mathrm{C}, \mathrm{Ca}$ \\
\hline Sclerolobium paniculatum Vogel & Fabaceae & 33 & MG, C \\
\hline Diospyros hispida A.DC. & Fabaceae & 32 & C \\
\hline Hymenaea stigonocarpa Mart. ex Hayne & Fabaceae & 32 & MG, C, Ca \\
\hline Hirtella ciliata Mart. \& Zucc. & Chrysobalanaceae & 28 & C \\
\hline Anacardium occidentale L. & Anacardiaceae & 21 & $\mathrm{C}, \mathrm{Ca}$ \\
\hline Psidium sp & Myrtaceae & 19 & C \\
\hline Byrsonima crassifolia (L.) Kunth & Malpighiaceae & 17 & $\mathrm{C}, \mathrm{Ca}$ \\
\hline Pouteria ramiflora (Mart.) Radlk. & Sapotaceae & 16 & MG, C \\
\hline Andira vermifuga Mart. ex Benth. & Fabaceae & 14 & MG, Ce, C \\
\hline Stryphnodendron coriaceum Benth. & Fabaceae & 13 & C \\
\hline Dimorphandra gardneriana Tul. & Fabaceae & 12 & $\mathrm{C}, \mathrm{Ca}$ \\
\hline Simarouba versicolor A.St.-Hil. & Simaroubaceae & 11 & MG, C, Ca \\
\hline Salacia elliptica A.St.-Hil. & Hippocrateaceae & 9 & MG, C \\
\hline Salvertia convallariodora (Mart. ex Schult.) G.Don & Vochysiaceae & 8 & $\mathrm{C}, \mathrm{Ca}$ \\
\hline Annona coriacea Mart. & Annonaceae & 7 & $\mathrm{Ce}, \mathrm{C}, \mathrm{Ca}$ \\
\hline Plathymenia reticulata Benth. & Fabaceae & 7 & $\mathrm{Ce}, \mathrm{C}$ \\
\hline Ouratea sp & Ochnaceae & 7 & C \\
\hline Agonandra brasiliensis Miers ex Benth. \& J.D.Hook. & Opiliaceae & 7 & MG, MS, C \\
\hline Combretum duarteanum Cambess. & Combretaceae & 6 & $\mathrm{MG}, \mathrm{Ce}, \mathrm{C}, \mathrm{Ca}$ \\
\hline Bowdichia virgilioides Kunth & Fabaceae & 6 & $\mathrm{MS}, \mathrm{Ce}, \mathrm{C}, \mathrm{Ca}$ \\
\hline Lafoensia vandelliana Cham. \& Schltdl & Lythraceae & 6 & MS, C \\
\hline Astronium fraxinifolium Schott ex Spreng. & Anacardiaceae & 5 & MG, C \\
\hline Combretum mellifluum Eichler & Combretaceae & 5 & MG, C \\
\hline Vatairea macrocarpa (Benth.) Ducke & Fabaceae & 5 & MG, Ce, C \\
\hline Eugenia dysenterica Mart. Ex DC. & Myrtaceae & 5 & $\mathrm{Ce}, \mathrm{C}$ \\
\hline Coccoloba sp & Polygonaceae & 5 & $\mathrm{C}$ \\
\hline Outras (19 espécies) & & 38 & \\
\hline
\end{tabular}


Tabela 2 - Variáveis ambientais encontradas para as parcelas amostradas no cerrado sensu stricto sobre Neossolo Quartzarênico da Chapada Grande Meridional, Piauí. MO: matéria orgânica; PST: Porcentagem de Sódio Trocável; CTC: capacidade de troca catiônica no pH atual do solo; T: capacidade de troca catiônica no pH 7; V: saturação de bases; m: saturação por alumínio; DS: desvio padrão; *: valores fora do intervalo de confiança para a média.

\begin{tabular}{|c|c|c|c|c|c|c|c|c|c|c|c|}
\hline $\begin{array}{c}\text { Variáveis / } \\
\text { Parcelas }\end{array}$ & 1 & 2 & 3 & 4 & 5 & 6 & 7 & 8 & 9 & 10 & Méc \\
\hline $\mathrm{pH}\left(\mathrm{H}^{2} 0\right)$ & 5,54 & 5,07 & 5,14 & 4,88 & 5,10 & 5,20 & 5,04 & 5,26 & 4,92 & 4,94 & $5,10(0,19)$ \\
\hline $\mathrm{Al}^{3+}\left(\mathrm{cmolc} \cdot \mathrm{kg}^{-1}\right)$ & 0,32 & 0,60 & 0,74 & 1,52 & 0,79 & 0,86 & 0,98 & 0,50 & 0,87 & 1,16 & $0,83(0,34)$ \\
\hline $\mathrm{H}+\mathrm{Al}\left(\mathrm{cmolc} . \mathrm{kg}^{-1}\right)$ & 1,60 & 1,72 & 1,88 & 2,34 & 2,38 & 2,14 & 2,38 & 1,32 & 2,04 & 2,12 & $1,99(0,36)$ \\
\hline $\mathrm{Cu}^{2+}\left(\mathrm{mg} \cdot \mathrm{L}^{-1}\right)$ & 0,50 & 0,60 & 0,50 & 0,50 & 0,60 & 0,50 & 0,50 & 0,60 & 0,60 & 0,70 & $0,56(0,07)$ \\
\hline $\mathrm{Fe}^{2+}\left(\mathrm{mg} \cdot \mathrm{L}^{-1}\right)$ & 48,50 & 51,6 & 35,2 & 80,3 & 53,6 & 59,2 & $154,6 *$ & 51,1 & 57,1 & 66,3 & $65,75(33,36)$ \\
\hline $\mathrm{Zn}^{2+}\left(\mathrm{mg} \cdot \mathrm{L}^{-1}\right)$ & 0,50 & 0,10 & 0 & 0,20 & 0,30 & 0,30 & 0,30 & 0,30 & 0,20 & 0,20 & $0,24(0,13)$ \\
\hline $\mathrm{Mn}^{2+}\left(\mathrm{mg} \cdot \mathrm{L}^{-1}\right)$ & 17,8 & 34 & $76 *$ & 10,3 & 17,4 & 24,4 & 6,8 & 46,2 & 21,6 & 17,6 & $27,21(20,56)$ \\
\hline $\mathrm{P}^{+}\left(\mathrm{mg} \cdot \mathrm{L}^{-1}\right)$ & 0,36 & 0,22 & 0,30 & 0,28 & 0,35 & 0,34 & 0,36 & 0,21 & 0,33 & 0,30 & $0,30(0,05)$ \\
\hline $\mathrm{Ca}^{2+}\left(\mathrm{cmolc} \mathrm{kg}^{-1}\right)$ & $0,58 *$ & 0,08 & 0,09 & 0,02 & 0,01 & 0,07 & 0,06 & 0,06 & 0,02 & 0,05 & $0,10(0,17)$ \\
\hline $\mathrm{Mg}^{2+}\left(\mathrm{cmolc} \cdot \mathrm{kg}^{-1}\right)$ & 0,33 & 0,15 & 0,15 & 0,11 & 0,18 & 0,16 & 0,26 & 0,16 & 0,07 & 0,18 & $0,17(0.07)$ \\
\hline $\mathrm{K}^{+}\left(\mathrm{cmolc} \cdot \mathrm{kg}^{-1}\right)$ & 1,31 & 1,08 & 1,13 & 1,29 & 1,00 & 1,26 & 1,55 & 1,13 & $0,54 *$ & 1,16 & $1,15(0,26)$ \\
\hline $\mathrm{Na}^{+}\left(\mathrm{cmolc} \cdot \mathrm{kg}^{-1}\right)$ & 0,03 & 0,00 & 0,02 & 0,03 & 0,00 & 0,00 & 0,03 & 0,00 & 0,00 & 0,03 & $0,02(0,02)$ \\
\hline Argila (\%) & 3,00 & 5,00 & 6,00 & 4,00 & 5,00 & 5,00 & & 6,00 & 5,00 & 4,00 & ,99) \\
\hline Silte (\%) & $1,00 *$ & 0,00 & 0,00 & 0,00 & 0,00 & 0,00 & 0,00 & 0,00 & 0,00 & 0,00 & $0,10(0,32)$ \\
\hline Areia grossa (\%) & 7,00 & 6,00 & 1,00 & 0,00 & 1,00 & 4,00 & 1,00 & 3,00 & 1,00 & 2,00 & $2,60(2,37)$ \\
\hline Areia fina (\%) & 89,00 & 89,0 & 93,0 & 96,0 & 94,0 & 91,0 & 93,00 & 91,0 & 94,00 & 94,0 & $92,40(2,32)$ \\
\hline MO (\%) & 0,36 & 0,22 & 0,30 & 0,28 & 0,35 & 0,34 & 0,36 & 0,21 & 0,33 & 0,30 & $0,31(0,05)$ \\
\hline CTC $\left(\mathrm{cmolc} . \mathrm{kg}^{-1}\right)$ & 2,58 & 1,91 & 2,13 & 2,97 & 1,98 & 2,35 & 2,88 & 1,85 & 1,50 & 2,58 & $2,27(0,48)$ \\
\hline PST (\%) & 1,30 & 0,00 & 0,77 & 1,13 & 0,00 & 0,00 & 1,16 & 0,00 & 0,00 & 1,29 & $0,66(0,61)$ \\
\hline $\mathrm{T}\left(\mathrm{cmolc} . \mathrm{kg}^{-1}\right)$ & 3,86 & 3,03 & 3,27 & 3,79 & 3,57 & 3,63 & 4,28 & 2,67 & 2,67 & 3,54 & $3,43(0,52)$ \\
\hline V $(\%)$ & 58,52 & 43,27 & 42,51 & 38,28 & 33,42 & 41,08 & 44,38 & 50,63 & 23,62 & 40,16 & $41,96(9,32)$ \\
\hline m (\%) & 12,42 & 31,38 & 34,74 & 51,15 & 39,81 & 36,56 & 34,04 & 26,98 & 57,96 & 44,92 & $36,67(12,74)$ \\
\hline Altitude (m) & 215,0 & 191,0 & 188,0 & 170,0 & 191,0 & 169,0 & 184,0 & 184,0 & 168,0 & 175,0 & $183,5(14,26)$ \\
\hline
\end{tabular}

Tabela 3 - Valores de correlação das variáveis nos primeiro e segundo eixos da Análise de Correspondência Canônica (CCA) para as amostras compostas de solos $(0-15 \mathrm{~cm}$ de profundidade) do cerrado sensu stricto sobre Neossolo Quartzarênico na Chapada Grande Meridional, Piauí.

\begin{tabular}{|c|c|c|}
\hline Variáveis & Eixo 1 & Eixo 2 \\
\hline $\mathrm{Na}^{+}$ & 0,6954 & 0,0793 \\
\hline $\mathrm{Al}^{3+}$ & 0,3969 & $-0,7485$ \\
\hline $\mathrm{K}^{+}$ & 0,3871 & 0,0599 \\
\hline $\mathrm{Zn}^{2+}$ & 0,2548 & 0,7017 \\
\hline $\mathrm{P}^{+}$ & 0,0901 & 0,4889 \\
\hline Areia grossa & 0,1296 & 0,7461 \\
\hline Altitude & 0,7240 & 0,3333 \\
\hline Auto-valor & 0,364 & 0,317 \\
\hline Variância cumulativa (\%) & 27,3 & 23,8 \\
\hline
\end{tabular}




\section{RELAÇÃo entre VegetAÇÃo e VARIÁVEIS AMBIENTAIS}

A CCA mostrou que as variáveis edáficas e altitude das parcelas explicaram mais da metade da variação na densidade e composição de espécies entre as parcelas, com o primeiro e segundo eixos explicando 51,1\% da variação dos dados (autovalores de 0,364 e 0,317 , respectivamente) e com uma correlação significativa em todos os eixos ( $\mathrm{F}=1,568$, $\mathrm{P}=0,03$ ) (Tabela 3). Os autovalores indicam um gradiente curto, onde poucas parcelas se destacaram pela presença de algum elemento, como $\mathrm{Al}^{3+}, \mathrm{P}^{+}, \mathrm{K}^{+}$ou $\mathrm{Zn}^{2+}$.

As variáveis ambientais que tiveram maior influência na vegetação foram: altitude, teores de $\mathrm{Na}^{+}, \mathrm{Al}^{3+}, \mathrm{Zn}^{2+}, \mathrm{P}^{+}, \mathrm{K}^{+}$e areia grossa, conforme podem ser visualizados na Figura 1 e na Tabela 3, mostrando correlações entre a estrutura da vegetação e o relevo, a textura e a fertilidade do solo. Esses fatores demonstram, de maneira indireta, a relação com o regime hídrico, pois a areia grossa e diferenças no relevo propiciam diferenças na drenagem de água no solo e corroboram Oliveira-Filho et al. (1989), que ao analisarem um gradiente de cerrado sobre solos arenosos na Chapada dos Guimarães, no Mato Grosso, associaram as variações da vegetação com as diferenças no regime de água no solo e declividade, devido à topografia e rochosidade do solo. Porém, esses autores não encontraram diferenças na fertilidade e textura do solo.

As variáveis mais correlacionadas com o primeiro eixo de ordenação foram teores de $\mathrm{Na}^{+}$e $\mathrm{K}^{+}$, enquanto o segundo eixo foi relacionado com os teores de $\mathrm{Al}^{3+}, \mathrm{Zn}^{2+}, \mathrm{P}^{+}$, areia grossa e altitude (Figura 1, Tabela 3). Dessa forma, o diagrama de ordenação mostra uma variação de salinidade no primeiro eixo e de fertilidade e textura no segundo eixo (Figura 1).

A P1, que apresentou os maiores valores para a fertilidade do solo e porcentagem de areia grossa, como evidenciado na caracterização do solo (Tabela 2), encontra-se na extremidade do segundo eixo (Figura 1), representando o extremo dos gradientes obtidos para as variáveis analisadas.

As variações no teor de $\mathrm{P}^{+}(0,21$ a 0,36 mg. $\left.\mathrm{L}^{-1}\right)$ são inferiores às comumente encon- tradas em áreas de Neossolo Quartzarênico sob cerrado. Na Chapada do Espigão Mestre do São Francisco, por exemplo, foram encontrados de 0,8 a 23,8 mg.L.-1 (Haridasan, 2001), exercendo grande influência sobre a vegetação. Os solos do cerrado possuem conhecida deficiência em $\mathrm{P}^{+}$(Lathwell \& Grove, 1986; Sousa \& Lobato 2004), sendo que mais de $90 \%$ desses possuem cerca de $0,4 \mathrm{mg} . \mathrm{L}^{-1}$ de fósforo disponível, enquanto os limites normalmente variam de 0,1 a 16,5 mg. $\mathrm{L}^{-1}$ (Furley \& Ratter, 1988). Os resultados obtidos no presente trabalho mostram que o teor de $\mathrm{P}^{+}$está próximo do limite inferior do que pode ser encontrado para solos do bioma e a pouca variação encontrada no gradiente do $\mathrm{P}^{+}$explica o baixo número de espécies mais correlacionadas com esta variável (Figura 1). As espécies mais correlacionadas com o $\mathrm{P}^{+}$foram Anacardium occidentale, além de ter ampla distribuição pelo Cerrado (Ratter et al., 2003, 2005; Castro et al., 1998), também é encontrada em matas de terra-firme, na Amazônia (Castro et al., 1998); e Dimorphandra gardneriana que possui uma distribuição mais restrita aos cerrados do Nordeste, porém também ocorre na Caatinga (Castro et al., 1998).

$\mathrm{O} \mathrm{K}^{+}$encontrado na Chapada Grande Meridional variou de 0,54 a 1,55 cmol. $\mathrm{kg}^{-1}$, acima dos valores comumente encontrados para o cerrado, entre 0,02 e 0,61 cmol. $\mathrm{kg}^{-1}$ (Furley \& Ratter, 1988; Lopes, 1983) mesmo em solos arenosos como na Chapada do Espigão Mestre, que variou de 0,027 a 0,162 cmol.kg-1 (Haridasan, 2001). As espécies mais correlacionadas com o $\mathrm{K}^{+}$foram Hirtella ciliata, Hymenaea stigonocarpa, Vatairea macrocarpa, Astronium fraxinifolium, Coccoloba sp e Andira vermifuga. Destas espécies, apenas Hirtella ciliata não ocorre em formações florestais (Tabela 1) e é característica dos cerrados do Nordeste (Bridgewater et al., 2005; Castro et al., 1998).

Em relação ao $\mathrm{Zn}^{2+}$, foram encontrados teores de 0 a $0,5 \mathrm{mg} . \mathrm{L}^{-1}$, com elevada variação entre as parcelas (Tabela 2). Esse elemento possui como nível crítico para as plantas 0,8 mg. $\mathrm{L}^{-1}$, sendo que $80 \%$ dos solos do cerrado encontram-se abaixo desse nível (Furley \& Ratter, 1988). As espécies mais relacionadas com o $\mathrm{Zn}^{2+}$ foram Vatairea macrocarpa, Astronium fraxinifolium e Coccoloba sp (Figura 1). O 


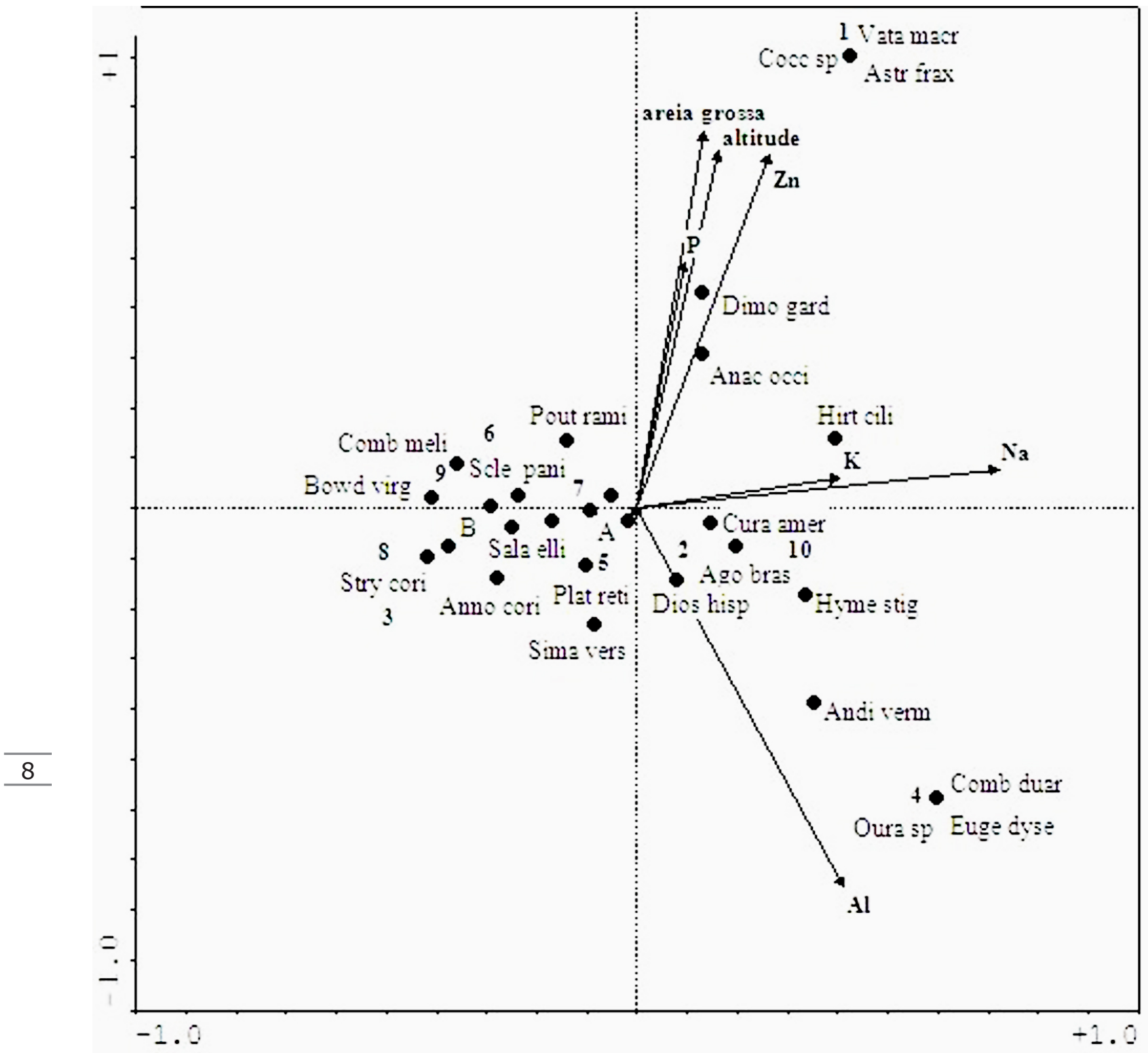

Figura 1 - Diagrama de ordenação produzido pela Análise de Correspondência Canônica, baseado na densidade absoluta de espécies, parcelas e propriedades do Neossolo Quartzarênico e altitude do cerrado sensu stricto da Chapada Grande Meridional, Piauí. Números de 1 a 10: parcelas amostradas; P: fósforo, Zn: zinco, K: potássio, Na: sódio, Al: alumínio; A: Qual gran, Salv conv, Park plat, Byrs crass; B: Qual parv, Psid $\mathrm{sp}$, Lafo vand. Códigos das espécies referem-se às quatro primeiras letras do binômio da nomenclatura botânica na Tabela 1.

gênero Coccoloba costuma ser abundante em florestas estacionais (Goodland \& Ferri, 1979). Astronium fraxinifolium só ocorreu na P1 e é indicadora de solos mesotróficos (Ratter et al., 2003, 2005; Castro et al., 1998) e também ocorre em outras áreas de cerrado do Piauí (Castro et al., 1998; Farias \& Castro, 2004) e matas de galeria (Felfili et al., 2001). Vatairea macrocarpa também ocorre nas matas de galeria no Brasil Central (Felfili et al., 2001) e cerradão (Mendonça et al., 1998). Essas espécies, também correlacionadas com o $\mathrm{K}^{+}$no primeiro eixo, indicam a sua ocorrência em ambientes com teor mais elevado de nutrientes. 
A maioria das espécies não respondeu à variação dos gradientes encontrados e ficaram concentradas no centro do diagrama, mostrando uma correlação negativa com o $\mathrm{Na}^{+}$no primeiro eixo (Figura 1). Isto sugere que, apesar de pouca quantidade de $\mathrm{Na}^{+}$(entre 0 a 0,03 cmol.kg-1) encontrada nos solos da Chapada Grande Meridional, foi suficiente para influenciar no estabelecimento de algumas espécies.

É provável que esse elemento seja transportado por chuvas e ventos com influências marítimas, como ocorre na Austrália (CRC, 1994). Esse argumento é suportado pelas já conhecidas elevadas concentrações de $\mathrm{Na}^{+}$encontrada no Parque Nacional de Sete Cidades (PNSC), no Piauí, distante aproximadamente $260 \mathrm{~km}$ ao norte da área estudada (Lindoso, 2008). O PNSC encontra-se a $140 \mathrm{~km}$ de distância do litoral e possuiu concentrações de $\mathrm{Na}^{+}$de 0,6 a $0,16 \mathrm{cmol} . \mathrm{kg}^{-1}$, apresentando solos com caráter solódico com porcentagem de sódio trocável (PST) entre 6\% e 15\% (Lindoso, 2008) de acordo com o Sistema Brasileiro de Classificação de Solos (Embrapa, 1999; IBGE, 2007). Já a Chapada Grande Meridional não possuiu nenhuma parcela com concentração de $\mathrm{Na}^{+}$suficiente para poder caracterizar o solo com caráter solódico (PST entre 0 a 1,30\%) e as áreas estudadas encontram-se mais distantes do litoral (aproximadamente $450 \mathrm{~km}$ ). Mesmo assim, $\mathrm{o} \mathrm{Na} \mathrm{Na}^{+}$exerce influência sobre a vegetação, como demonstrado na ordenação (Figura 1) e, portanto, merece maior atenção, uma vez que esse elemento não é normalmente averiguado em estudos no bioma Cerrado.

As espécies mais correlacionadas com o $\mathrm{Na}^{+}$(Figura 1) foram Eugenia dysenterica, Combretum duarteanum e Ouratea sp, que também apresentaram correlação negativa com os nutrientes $\mathrm{Zn}^{2+}$ e $\mathrm{P}^{+}$e foram positivamente correlacionadas com o $\mathrm{Al}^{3+}$. Dessa forma, essas espécies podem ser sugeridas para a recuperação de áreas na região com solos distróficos e álicos.

Já o teor de $\mathrm{Al}^{3+}$, que variou de 0,32 a 1,52 cmol. $\mathrm{kg}^{-1}$, foi maior na P4, a qual também obteve a maior densidade (134 indivíduos). De acordo com Goodland (1971) e Goodland \& Ferri (1979), os cerrados apresentam um oligotrofismo aluminotóxico, onde há influência do caráter tóxico do alumínio sobre a vegetação, o que determina um gradiente de diminuição da fitomassa com maior concentração deste elemento e menor fertilidade nos solos, o que não foi corroborado neste estudo $(P=0,30)$, assim como em outros trabalhos realizados em cerrado s.s. ou outras fisionomias, como o cerradão (Haridasan, 1992; Marimon et al., 2001; Marimon-Junior \& Haridasan, 2005; Ruggiero et al., 2002). Essa parcela foi a que teve o $\mathrm{pH}$ mais baixo, relacionado com o maior teor de $\mathrm{Al}^{3+}(\mathrm{r}=0,826, \mathrm{P}=0,003)$, já que este é um componente que contribui para a acidez do solo (Brady \& Weil, 2002; Goedert, 1987).

A P1 foi a que obteve maior $\mathrm{pH}(5,54)$, os maiores teores de $\mathrm{Ca}^{2+}\left(0,58 \mathrm{cmol}^{2} \mathrm{~kg}^{-1}\right), \mathrm{P}^{+}$ $\left(0,36 \mathrm{cmol} \cdot \mathrm{kg}^{-1}\right)$ e $\mathrm{Mg}^{2+}\left(0,33 \mathrm{cmol} \cdot \mathrm{kg}^{-1}\right)$, maior saturação por bases $(58,52 \%)$ e menor saturação por $\mathrm{Al}^{3+}(12,41 \%)$, além de maior porcentagem de areia grossa (7\%). Essa parcela foi a que se localizou mais ao norte (no município de Regeneração) e com maior altitude (215 m). A menor acidez encontrada nesse solo pode estar indiretamente relacionada com a presença de silte (Tabela 2), apresentando uma alta correlação com o pH ( $\mathrm{r}=0,77, \mathrm{P}=0,008)$ e teor de bases como $\mathrm{Ca}^{2+}(\mathrm{r}=0,98, \mathrm{P}<0,001)$.

Os resultados mostram que mesmo plantas nativas, adaptadas à acidez e à baixa disponibilidade de nutrientes respondem a pequenas diferenças na fertilidade, assim como à presença de $\mathrm{Na}^{+}$no solo. Estudos de ecofisiologia em relação aos níveis críticos de nutrientes para plantas nativas, assim como fluxo de água no solo e sua relação com a vegetação poderão elucidar os padrões aqui encontrados.

\section{Conclusões}

Os resultados do presente trabalham corroboram com a hipótese levantada de que o desenvolvimento da vegetação lenhosa responde a pequenas variações nos atributos edáficos. Os solos analisados na Chapada Grande Meridional revelaram-se com baixa variação de fertilidade e, consequentemente, com pouca diferenciação florística das espécies de cerrado s.s. encontradas sobre Neossolo Quartzarênico. Mesmo plantas nativas, adaptadas à acidez e baixa disponibilidade de nutrientes no solo, respondem a pequenas diferenças encontradas na concentração de nutrientes como 
teores de $\mathrm{Na}^{+}, \mathrm{K}^{+}, \mathrm{Al}^{3+}, \mathrm{P}^{+}, \mathrm{Zn}^{2+}$, na porcentagem de areia grossa, na altitude e na salinidade do solo. Estas pequenas diferenças são suficientes para determinar diferentes densidades das espécies, promovendo uma elevada diversidade beta entre as parcelas analisadas.

A concentração de sódio parece influenciar a distribuição da vegetação e deve estar correlacionada com a distância do mar. Apesar das concentrações encontradas não serem suficientes para caracterizar os solos da Chapada Grande Meridional como solódicos (PST $\leq 6 \%$ ), os resultados mostram que a influência deste elemento sobre vegetação de cerrado s.s. pode ser mais importante do que normalmente se supõe, merecendo maior atenção, uma vez que esse elemento não é normalmente averiguado em estudos no bioma Cerrado.

\section{Agradecimentos}

Agradecemos à equipe de campo e do BioTEN (UFPI/CCN/Biologia) que apoiaram o desenvolvimento da pesquisa na Chapada Grande Meridional, ao Laboratório de Química Analítica da EMBRAPA/CPAC pela análise dos solos, ao CNPq-PELD - Sítio 10 e ao Programa de Pós Graduação em Ecologia UnB pelo auxílio e ao CNPq pela concessão da bolsa de mestrado.

\section{REFERÊNCIAS}

Aguiar, R.B. \& R.B.C. Gomes. 2004. Projeto cadastro de fontes de abastecimento por água subterrânea, Estado do Piauí. CPRM - Serviço Geológico do Brasil, Fortaleza, 21 p.

Andrade Júnior, A.S., E.A. Bastos, A.H.C. Barros, C.O. Silva \& A.A.N. Gomes. 2004. Classificação climática do estado do Piauí. Documentos 86. Embrapa MeioNorte, Teresina, 86 p.

Brady, N.C. \& R.R. Weil. 2002. The nature and properties of soils. Prentice Hall, New Jersey, $960 \mathrm{p}$.

Bridgewater, S.; J.A. Ratter \& J.F. Ribeiro. 2005. Biogeographic patterns, - -diversity and dominance in the Cerrado biome of Brazil. Biod. Cons. 13: 2295-2318.
Castro, A.A.J.F \& F.R. Martins. 1999. Cerrados do Brasil e do Nordeste: caracterização, área de ocupação e considerações sobre a sua fitodiversidade. Pesq. Foco 7: 147-178.

Castro, A.A.J.F., F.R. Martins \& A.G. Fernandes. 1998. The woody flora of cerrado vegetation in the state of Piauí, northeastern Brazil. Edinb. J. Bot. 55: 455-472.

CRC - Cooperative Research Centre for Soil \& Land Management. 1994. Technical Note: introduction to soil sodicity. University of Adelaide / Csiro division of soils / South Australian Research and Development Institute, $4 \mathrm{p}$.

Day, P.R. 1965. Particle fractionation and particlesize analysis, p. 545-567. In: C.A. Black (Ed), Methods of Soil Analysis. Madison, Wisconsin, American Society of Agronomy.

Eiten, G. 1972. The cerrado vegetation of Brazil. Bot. Rev. 38: 201-304.

Embrapa. 1997. Manual de Métodos de Análise de Solo. Centro Nacional de Pesquisa de Solos, Rio de Janeiro, 212 p.

Embrapa. 1999. Sistema Brasileiro de Classificação de Solos. Centro Nacional de Pesquisa de Solos, Rio de Janeiro, $412 \mathrm{p}$.

Embrapa. 2005. Manual de laboratórios: solo, água, nutrição animal e alimentos (A.R.A Nogueira e G.B. Souza, eds.) Embrapa Pecuária Sudeste, São Carlos, 313p.

Farias, R.R.S \& A.A.J.F. Castro. 2004. Fitossociologia de trechos da vegetação do complexo de Campo Maior, Campo Maior, PI, Brasil. Acta Bot. Bras. 18: 949-963.

Felfili, J.M., A.V. Rezende \& M.C. Silva Júnior. 2007. Biogeografia do bioma Cerrado: vegetação e solos da Chapada dos Veadeiros. Editora Universidade de Brasília/Finatec, Brasília, 256p.

Felfili, J.M. \& M.C. Silva Júnior. 1993. A comparative study of cerrado (sensu stricto) vegetation in central Brazil. J. Trop. Biol.9: 277-289.

Felfili, J.M. \& M.C. Silva Júnior. 2001. Biogeografia do bioma Cerrado: estudo fitofisionômico na Chapada do Espigão Mestre do São Francisco. UnB/Departamento de Engenharia Florestal, Brasília, 152 p.

Felfili, J.M. \& M.C. Silva Júnior. 2005. Diversidade alfa e beta no cerrado sensu stricto, Distrito Federal, Goiás, Minas Gerais 
e Bahia, p.143-154. In: A. Scariot, J.C. Sousa-Silva \& J.M. Felfili (Eds), Cerrado: ecologia, biodiversidade e conservação. Ministério do Meio Ambiente, Brasília.

Felfili, J.M., R.C. Mendonça, B.M.T. Walter, M.C. Silva Júnior, M.G.G. Nóbrega, C.W. Fagg, A.C. Sevilha \& M.A. Silva. 2001. Flora fanerogâmica das matas de galeria e ciliares do Brasil central, p.195263. In: J.F. Ribeiro, C.E.L. Fonseca \& J.C. Souza-Silva (Eds), Cerrado: caracterização e recuperação de matas de galeria. Embrapa Cerrados, Planaltina.

Felfili, J.M., M.C. Silva Júnior, A.C. Sevilha, C.W. Fagg, B.M.T.W. Walter, P.E. Nogueira \& A.V. Rezende. 2004. Diversity, floristic and structural patterns of Cerrado vegetation in central Brazil. Pl. Ecol. 175: 37-46.

Felfili, M.C. \& J.M. Felfili. 2001. Diversidade alfa e beta no cerrado sensu stricto da Chapada Pratinha, Brasil. Acta Bot. Bras. 15: 243-254.

Furley, P.A \& J.A. Ratter. 1988. Soil resources and plant communities of the central brazilian Cerrado and their development. J. Biogeogr. 15: 97-108.

Goedert, W.J. 1987. Solos dos cerrados: tecnologias e estratégias de manejo. Nobel/Embrapa-CPAC, São Paulo / Brasília, 422 p.

Goodland, R. \& M.G. Ferri. 1979. Ecologia do Cerrado. Ed. Itatiaia, Belo Horizonte, 193 p.

Goodland, R. \& R. Pollard. 1973. The brazilian cerrado vegetation: a fertility gradient. J. Ecol. 61: 219-224.

Goodland, R. 1971. A physiognomic analysis of the cerrado vegetation of central Brazil. J. Ecol. 59: 411-419.

Haridasan, M. 1992. Observation on soils, foliar nutrients concentrations, and floristic, composition of cerrado and cerradão communities in central Brazil, p.174-184. In: J. Proctor, J.A. Ratter \& P.A. Furley (Eds), The nature and dynamics of forest-savanna boundaries. Chapman \& Hall, London.

Haridasan, M. 2001. Solos, p.12-17. In: J.M. Felfili \& M.C. Silva Júnior (Eds), Biogeografia do bioma Cerrado: estudo fitofisionômico na Chapada do Espigão Mestre do São Francisco. UnB/Departamento de Engenharia Florestal, Brasília.
Haridasan, M. 2007. Solos, p. 25-43. In: J.M. Felfili, A.V. Rezende \& M.C. Silva Júnior (Eds), Biogeografia do bioma Cerrado: vegetação e solos da Chapada dos Veadeiros. Editora Universidade de Brasília/ Finatec, Brasília.

IBGE - Instituto Brasileiro de Geografia e Estatística. 2007. Manual técnico de pedologia. IBGE, Rio de Janeiro 326 p.

Lathwell, D.J. \& T.L. Grove. 1986. Soil - plant relationships in the tropics. Annu. Rev. Ecol. Systemat. 17: 1-16.

Leps, J. \& P. Smilauer. 2003. Multivariate analysis of ecological data using Canoco. University of Cambridge, United Kingdon, $296 \mathrm{p}$.

Lima, MG. \& H.F. Assunção. 2002. Estimativa da temperatura no ar no Piauí. Edufpi, Teresina, $48 \mathrm{p}$.

Lindoso, G.S \& J.M. Felfili. 2007. Características florísticas e estruturais de cerrado sensu stricto em Neossolo Quartzarênico. Rev. Bras. Bioc. 5: 102-104.

Lindoso, G.S., J.M. Felifili, J.M. da Costa \& A.A.J.F. Castro. 2009. Diversidade e estrutura do cerrado sensu stricto sobre areia (Neossolo Quartzarênico) na Chapada Grande Meridional, Piauí. Rev. Biol. Neotrop. 6:45-61.

Lindoso, G.S. 2008. Cerrado sensu stricto sobre Neossolo Quartzarênico: Fitogeografia e Conservação. Dissertação de Mestrado. Programa de Pós-graduação em Ecologia, Universidade de Brasília, Brasília, 186 p.

Lopes, A.S. 1983. Solos sob cerrado: características, propriedades e manejo. Instituto de Potassa \& Fosfato, Instituto da Potassa, Piracicaba.

Luz, M.J.S., G.B. Ferreira, \& J.R.C. Bezerra. 2002. Adubação e correção do solo: procedimentos a serem adotados em função dos resultados da análise do solo. Embrapa Circular Técnica 63, Embrapa, Ministério de Agricultura e Abastecimento, Campina Grande, 32 p.

Marimon-Junior, B.H. \& M. Haridasan. 2005. Comparação da vegetação arbórea e características edáficas de um cerradão e um cerrado sensu stricto em áreas adjacentes sobre solo distrófico no leste de Mato Grosso, Brasil. Acta Bot. Bras. 19: 913-926. 
Marimon, B.S., J.M. Felfili \& M. Haridasan. 2001. Studies in monodominant forests in eastern Mato Grosso, Brazil: i. A forest of Brosimun rubescens taub. Edinb. J. Bot. 58: 123-137.

Mendonça, R.C., J.M. Felfili, B.M.T. Walter, M.C. Silva Júnior, A.V. Rezende, T.S. Filgueiras \& P.N. Ernani. 1998. Flora vascular do Cerrado, p. 289-556. In: S.M. Sano \& S.P. Almeida (Orgs.), Cerrado: ambiente e flora Embrapa Cerrados, Planaltina.

Oliveira-filho, A.T. \& J.A. Ratter. 2002. Vegetation physiognomies and woody flora of Cerrado biome, p: 91-120. In: P.S. Oliveira \& R.J. Marquis (Orgs.), The cerrados of Brazil: ecology and natural history of a neotropical savanna. Columbia University Press, New York.

Oliveira-filho, A.T., G.J. Shepherd, F.R. Martins \& W.H. Stubblebine. 1989. Environmental factors affecting physiognomics and floristic variation in an area of Cerrado in central Brazil. J. Trop. Ecol. 5: 413-431.

Ratter, J.A., S. Bridgewater, J.F. Ribeiro. 2003. Analysis of the floristic composition of the brazilian cerrado vegetation III: comparison of the wood vegetation of 376 areas. Edinb. J. Bot. 60: 57-109.

Ratter, J.A., S. Bridgewater, J.F. Ribeiro. 2005. Biodiversity patterns of the woody vegetation of the brazilian cerrados, p.3158. In: R.T. Pennington, G.P. Lewis \& J.A. Ratter (Eds), Neotropical savannas and dry forests: diversity, biogeography and conservation. Oxford, CRC Press.

Ratter, J.A., J.F. Ribeiro \& S. Bridgewater. 1997. The brazilian Cerrado vegetation and threats to its biodiversity. Ann. Bot. 80: 223-230.

Reatto, A. \& E.S. Martins. 2005. Classes de solo em relação aos controles da paisagem do bioma Cerrado, p.49-59. In: A. Scariot, J.C. Souza-Silva \& J.M. Felfili (Eds), Cerrado: ecologia, biodiversidade e conservação. Ministério do Meio Ambiente, Brasília.

Reatto, A., J.R. Correia \& S.T. Spera. 1998. Solos do bioma Cerrado: aspectos pedológicos, p. 47-86. In: S.M. Sano \& S.P. Almeida, (Eds). Cerrado: ambiente e flora Embrapa Cerrados, Planaltina.
Rodal, M.J.N., A.C.B. Lins e Silva, L.M. Pessoa \& A.D.C. Cavalcanti. 2005. Vegetação e flora fanerogâmica da área de Betânia, Pernambuco, p.139-166. In: F.S. Araújo, M.J.N. Rodal \& M.R.V. Barbosa (Eds), Análise das variações da biodiversidade do bioma Caatinga: suporte a estratégias regionais de conservação. Ministério do Meio Ambiente, Brasília.

Ruggiero, P.G.C., M.A. Batalha, V.R. Pivello \& S.T. Meirelles. 2002. Soil-vegetation relationships in Cerrado (Brazilian savanna) and semideciduous forest, southeastern Brazil. Plant Ecol 160:1-16.

Silva, J.F., M.R. Fariñas, J.M. Felfili \& C.A. Klink. 2006. Spatial heterogeneity, land use and conservation in the cerrado region of Brazil. J. Biogeogr. 33: 536-548.

Silva, L.C.R., L. Sternberg, M. Haridasan, W.A. Hoffmann, F. Miralles-Wilhelm \& A.C. Franco. 2008. Expansion of gallery forests into central brazilian savannas. Global Change Biology 14:1-11.

Sousa, D.M.G. \& E. Lobato. 2004. Cerrado correção do solo e adubação. Embrapa Informações Tecnológicas, Brasília. 416 p.

Sousa, D.M.G. \& E. Lobato. 2005. Areias quartzosas / neossolo quartzarênico. www.embrapa.com.br.

Ter Braak, C.J.F. \& P. Smilauer. 1999. Canoco for Windows Version 4.02. Centre for Biometry Wageningen, Wageningen.

Ter Braak, C.J.F. 1986. Canonical correspondence analysis: a new eigenvector technique for multivariate direct gradient analysis. Ecol. 67:1167-1179.

Thornthwaite, C.W. \& J.R. Mather. 1955. The water balance. In publications in climatology. Drexel Institute of Technology, New Jersey. 104 p.

Walkley, A. \& I.A. Black. 1934. An examination of the Degtjareff method for determining soil organic matter and a proposed modification of the chronic tritation method. Soil Science 37: 29-38.

Recebido em 28/III/2011 Aceito em 01/XII/2011 